J. Austral. Math. Soc. 20 (Series A) (1975), 483-503.

\title{
TWENTY-STEP ALGORITHM FOR DETERMINING THE ASYMPTOTIC NUMBER OF TREES OF VARIOUS SPECIES*
}

\author{
FRANK HARARY, ROBERT W. ROBINSON and ALLEN J. SCHWENK
}

(Received 5 November 1974)

\section{Dedicated to E. M. Palmer on his fortieth birthday}

\section{Introduction}

The technique for finding the asymptotic number of unlabeled trees of various sorts was developed by Pólya (1937) and perfected by Otter (1948). Modern presentations are available in the book of Harary and Palmer (1973; Chapter 9), and in the paper of Bender (to appear).

An exposition of the basic method is here developed in the form of a 20 step algorithm, which should facilitate the finding of asymptotic formulas for different kinds of trees. These 20 steps are presented in Section 2, and methods of justifying the steps are supplied in Section 3. In Sections 4, 5 and 6, the algorithm is applied to finding asymptotic values for the number of identity trees, homeomorphically irreducible trees, and a class of blocks with tree-like properties. The first two of these species were enumerated by Harary and Prins (1959) and the third is easily done. However, no asymptotic analyses have been given previously.

For the purpose of the discussion in Sections 2 and 3, a hypothetical class of trees is posed, of which there are $S_{n}$ planted trees on $n+1$ points (including the root which is an endpoint; hence there are $n$ lines) and $s_{n}$ unrooted trees on $n$ points. We let $S(x)$ and $s(x)$ be the ordinary generating functions

$$
\begin{aligned}
& S(x)=\sum_{i=1}^{\infty} S_{i} x^{i}, \\
& S(x)=\sum_{i=1}^{\infty} s_{i} x^{i} .
\end{aligned}
$$

* Supported by the Air Force Office of Scientific Research. 
It is useful for the asymptotic analysis to regard $S(x)$ and $s(x)$ as power series in the complex variable $x$. Let $\sigma$ be the radius of convergence of the series $S(x)$.

Just two assumptions are made at the outset for our hypothetical class of trees. The first is that there are infinitely many trees in the class. The second is that no natural number except 1 divides every $n$ for which $S_{n}>0$. If the latter failed, we could proceed by expressing the generating functions in terms of the variable $y=x^{m}$ where $m$ is the greatest common divisor of all $n$ for which $S_{n}>0$.

\section{The twenty steps}

In this section we merely list the 20 steps which must be taken in order to obtain an asymptotic evaluation for any given species of trees. Only enough details are mentioned here to provide an understanding and flavor for each step. Indications of the procedure and the justification of each step are given in the next section.

STEP 1. Develop a functional relation giving $S(x)$ in terms of $S(x), S\left(x^{2}\right)$, $S\left(x^{3}\right), \cdots$. This is equivalent to a recurrence relation satisfied by the numbers $S_{n}$.

STEP 2. Develop a second functional relation giving $s(x)$ in terms of $S(x)$ and $S\left(x^{2}\right)$. This will correspond to an identity giving the numbers $s_{n}$ in terms of the $S_{n}$.

STEP 3. Verify that the radius of convergence $\sigma$ of $S(x)$ satisfies

$$
0<\sigma \leqq 1
$$

Step 4. Prove that $S(\sigma)<\infty$, and so $\sigma<1$.

STEP 5. In the functional relation for $S(x)$ obtained in Step 1, replace each occurrence of $S(x)$ by $y$ to produce a function $F(x, y)$ analytic for all $y$ and for all $x$ with $|x|<\sigma^{1 / 2}$ such that

for all $|x|<\sigma$.

$$
F(x, S(x))=0
$$

SteP 6. Observe that $F(x, S(x))=0$ for all $x$ with $|x|=\sigma$.

STEP 7. Take the partial derivative with respect to $y$ of the function $F(x, y)$ to obtain $F_{y}(x, y)$. First substitute $y=S(x)$ in this last expression and then substitute $x=\sigma$. Argue analytically that

$$
F_{y}(\sigma, S(\sigma))=0 .
$$

STEP 8. Use the result of Step 7 to evaluate $S(\sigma)$ in terms of $\sigma$. It often transpires that $S(\sigma)=1$. 
Step 9. Use the equation $F(\sigma, S(\sigma))=0$ (from Step 6) and the result of Step 8 to find equations from which the value of $\sigma$ may be calculated to any desired degree of accuracy.

STEP 10. Observe that we must have $F_{y}(x, S(x)) \neq 0$ for all $x$ such that $|x|=\sigma$ except $x=\sigma$.

SteP 11. Deduce that $\sigma$ is the unique singularity of $S(x)$ on its circle of convergence.

STEP 12. Using the results of Steps 3 and 7, verify that

$$
F_{\mathrm{yy}}(\sigma, S(\sigma)) \neq 0 \text {. }
$$

STEP 13. Deduce that $\sigma$ is a branch point of order 2 for $S(x)$.

STEP 14. By Step 13 and the relation from Step 2 giving $s(x)$ in terms of $S(x)$ and $S\left(x^{2}\right)$, note that $S(x)$ and $s(x)$ have expansions of the form

$$
\begin{aligned}
& S(x)=S(\sigma)-b_{1}(\sigma-x)^{1 / 2}+b_{2}(\sigma-x)+b_{3}(\sigma-x)^{3 / 2}+\cdots, \\
& S(x)=a_{0}+a_{1}(\sigma-x)^{1 / 2}+a_{2}(\sigma-x)+a_{3}(\sigma-x)^{3 / 2}+\cdots,
\end{aligned}
$$

for some $b_{1} b_{2}, b_{3}, \cdots$ and $a_{0}, a_{1}, a_{2}, \cdots$, and these series converge absolutely in some neighborhood of $x=\sigma$.

STEP 15. From Step 14, conclude that $b_{1}^{2} / 2=\lim _{x \rightarrow-r} S^{\prime}(x)(S(\sigma)-S(x))$.

STEP 16. Use Step 15 and the functional equation (Step 1) for $S(x)$ to find an equation giving $b_{1}$ in terms of $\sigma$ and the numbers $S_{1}, S_{2}, S_{3}, \cdots$. Then $b_{1}$ can be calculated to any desired degree of accuracy in view of Step 9. Show that $b_{1}>0$.

STEP 17. Deduce from $b_{1}>0$ and the form of $S(x)$ given in Step 14 that

$$
S_{n} \sim \frac{b_{1}}{2}\left(\frac{\sigma}{\pi}\right)^{1 / 2} n^{-3 / 2} \sigma^{-n} .
$$

STEP 18. Use the functional equation for $s(x)$ from Step 2 to deduce that $a_{1}=\mathbf{0}$.

STEP 19. On the basis of the functional equation for $s(x)$ and the result of Step 18, find an equation for $a_{3}$ similar to the one found in Step 16 for $b_{1}$. Show that $a_{3}>0$.

STEP 20. Deduce from $a_{1}=0<a_{3}$ and the form of $s(x)$ given in Step 14 that

$$
s_{n} \sim \frac{3 a_{3}}{4}\left(\frac{\sigma^{3}}{\pi}\right)^{1 / 2} n^{-5 / 2} \sigma^{-n} .
$$




\section{Justifying the steps in the algorithm}

In this section, we indicate some justification for each of the 20 steps presented above, insofar as this justification is independent of the hypothetical species of tree counted by $S(x)$ and $s(x)$.

STEP 1. The specific functional equation satisfied by $S(x)$ must be deduced from the structure of the species of tree which is being counted. (Examples are given in Harary and Prins (1959) and in Harary and Palmer (1973; pages 59-68).) However the starting point is always to break each planted tree into smaller ones, which are branches at the point adjacent to the root endpoint. Pólya's Hauptsatz (1937; page 163) is often useful for putting the combinatorial relation entirely in terms of $S(x), S\left(x^{2}\right), S\left(x^{3}\right), \cdots$.

STEP 2. Otter's dissimilarity characteristic (1948; page 588 ) can usually be applied to express $s(x)$ in terms of $S(x)$ and $S\left(x^{2}\right)$. This will involve analysing line-rooted trees of the species, and those with a symmetric line, in terms of the pair of planted trees obtained when that line is cut and each cut end is terminated by a root point.

In most cases the functional relations obtained in this step are sufficiently manageable that the computations required later can be carried out explicitly.

STEP 3. The upper bound, $\sigma \leqq 1$, follows from the fact that $S_{n} \geqq 1$ for infinitely many $n$. To establish the lower bound $0<\sigma$, compare $S(x)$ with some larger generating function having a known positive radius of convergence. The comparison series counts a wider species of trees. This is sometimes accomplished by embedding the given trees in the plane.

STEP 4. To obtain $S(\sigma)<x$, it is necessary to study the functional equation satisfied by $S(x)$ as $x$ approaches $\sigma$ from below. The key idea is to rewrite this equation so that one side grows as a polynomial function of $S(x)$, while the other side grows exponentially in $S(x)$. If $S(x)$ were unbounded as $x$ approaches $\sigma$, the exponential side would have to exceed the polynomial side for values of $x$ sufficiently close to $\sigma$. From $S(\sigma)<x$ and $S_{n} \geqq 1$ infinitely often, it is seen at once that $\sigma<1$.

STEP 5. To see that the function $F(x, y)$ obtained in this step is analytic at any $\left(x_{0}, y_{0}\right)$ with $\left|x_{0}\right|<\sigma^{1 / 2}$, consider $S\left(x^{2}\right), S\left(x^{3}\right), \cdots$ as given functions analytic for $|x|<\sigma^{1 / 2},|x|<\sigma^{1 / 3}, \cdots$ where $\sigma^{1 / 2}<\sigma^{1 / 3}<\cdots$. That $F(x, S(x))=0$ for $|x|<\sigma$ is a restatement of the functional equation satisfied by $S(x)$, found in Step 1.

STEP 6. Since $S(\sigma)<x$ and every $S_{i} \geqq 0$, one knows of course that $S(x)$ is finite for all $x$ on the circle $|x|=\sigma$. To extend the relation $F(x, S(x))=0$ from 
the interior of this circle to the circumference, appeal can be made to Abel's theorem on continuity up to the circle of convergence; see Whittaker and Watson (1943; page 57).

STEP 7. We need to verify the equation $F_{y}(\sigma, S(\sigma))=0$. The justification revolves around the fact that $\sigma$ must be a singularity of $S(x)$. This follows at once from Pringsheim's Theorem (see Hille (1959; page 133)) because $S_{i} \geqq 0$ for all $i$. Assume now that $F_{y}(\sigma, S(\sigma)) \neq 0$. Then the inverse function theorem (see Hille (1959; page 269)) implies that for each $x$ in some neighborhood of $\sigma$, there is just one number $\phi(x)$ for which $F(x, \phi(x))=0$, and $\phi(x)$ is analytic in this neighborhood. But then by Step $5, \phi(x)=S(x)$ for all $x$ in this neighborhood for which $|x| \leqq \sigma$. Thus $\phi(x)$ would be a continuation of $S(x)$ analytic at $x=\sigma$, contrary to the fact that $\sigma$ is a singularity of $S(x)$. Thus in fact we must have $F_{y}(\sigma, S(\sigma))=0$.

STEP 8. This step is usually handled routinely. For in general the form of $F(x, y)$ is such that $S(\sigma)$ can be determined explicitly from the equation $F_{y}(\sigma, S(\sigma))=0$.

STEP 9. Substituting into the equation $F(\sigma, S(\sigma))=0$ the expression for $S(\sigma)$ in terms of $\sigma$ given in Step 8, one can efficiently approximate $\sigma$ to any desired degree of accuracy.

STEP 10. The form of $F_{y}(x, S(x))$ is a linear function of $S(x)$ and we have just shown that $F_{y}(\sigma, S(\sigma))=0$. Thus, to conclude that $F_{y}(x, S(x)) \neq 0$ for $|x| \leqq \sigma$, but $x \neq \sigma$, it will suffice to show that for such $x$ we have $|S(x)|<S(\sigma)$.

The triangle inequality assures us that for $|x| \leqq \sigma$,

$$
S(x) \leqq S(\sigma),
$$

with equality only if every nonzero term in the power series $S(x)$ is in fact real and positive. Hence we have verified the desired strict inequality unless $x^{n}=\sigma^{n}$ whenever $S_{n}>0$. But we were careful at the outset to define $S(x)$ so that these values of $n$ have 1 as their greatest common divisor and so an appropriate linear combination of values of $n$ with $x^{n}=\sigma^{n}$ yields in fact $x=\sigma$. But this is the single value of $x$ excluded from consideration in this step, and so we have verified that $F_{y}(x, S(x)) \neq 0$ for $|x| \leqq \sigma$ but $x \neq \sigma$.

STEP 11. In Step 7, it was seen that $\sigma$ is a singularity of $S(x)$. For any other point on the circle of convergence, the result of Step 10 allows the implicit function theorem to be applied as in Step 7, showing that $S(x)$ is analytic there.

STEP 12. The inequality $F_{y y}(\sigma, S(\sigma)) \neq 0$ follows from an explicit second differentiation of $F_{y}(x, y)$ to obtain 


$$
F_{y y}(x, y)=F_{y}(x, y)+c
$$

where $c$ is a nonzero constant, usually $c=1$. Consequently the equality $F_{y}(\sigma, S(\sigma))=0$ implies that

$$
F_{y y}(x, y)=c \neq 0 \text {. }
$$

STEP 13. That $\sigma$ is a branch point of order 2 for $S(x)$ follows from the implicit function theorem, in view of the equations

$$
F_{y}(\sigma, S(\sigma))=0 \quad F_{y y}(\sigma, S(\sigma)) \neq 0 .
$$

Bender (to appear) goes into considerable detail on this point.

STEP 14. $S(x)$ is regular as a function of $(\sigma-x)^{1 / 2}$ near $x=0$ because $\sigma$ is a branch point of order 2 for $S(x)$, justifying the validity of an expansion in powers of $(\sigma-x)^{1 / 2}$. The relation found in Step 2 for $s(x)$ in terms of $S(x)$ and $S\left(x^{2}\right)$ provides at once a similar expansion for $s(x)$.

STEP 15. The equation

$$
b_{1}^{2} / 2=\lim _{x \rightarrow-\sigma} S^{\prime}(x)(S(\sigma)-S(x))
$$

is a simple computation from the series expansion of Step 14, viz.

$$
S(x)=S(\sigma)-b_{1}(\sigma-x)^{1 / 2}+b_{2}(\sigma-x)+\cdots .
$$

STEP 16. By considering the equation

$$
0=\lim _{x \rightarrow-\sigma} \frac{d}{d x} F(x, S(x))
$$

it will be found that

$$
\lim _{x \rightarrow-\sigma} S^{\prime}(x)(S(\sigma)-S(x))
$$

can be evaluated explicitly in terms of a series in $\sigma$ involving the numbers $S_{0}, S_{1}, S_{2}, \cdots$ in the coefficients. This is easily used to evaluate $b_{1}^{2}$ in view of the procedure available from Step 9 for evaluating $\sigma$ to any desired degree of accuracy. It will be obvious that $b_{1} \neq 0$. The sign of $b_{1}$ determines whether the expansion about $x=\sigma$ agrees with the expansion about $x=0$ where the domains overlap, without having to circle the point $x=\sigma$. For the next step, $b$, must be positive.

STEP 17. The asymptotic behavior of $S_{n}$ is deduced from the useful lemma of Pólya presented below, assuming that $b_{1}>0$ in the expansion of Step 14. Collecting alternate terms we have

$$
S(x)=(\sigma-x)^{1 / 2} A(x)+B(x)
$$


where $A(x)$ and $B(x)$ are analytic at $x=\sigma$ and $A(\sigma)=-b_{1}$. Use is made of the fact that $\Gamma(-1 / 2)=-2 \pi^{1 / 2}$.

Lemma (Pólya (1937; page 240)). Let the power series

$$
f(x)=a_{0}+a_{1} x+a_{2} x^{2}+\cdots
$$

have the finite radius of convergence $\alpha>0$, with $x=\alpha$ the sole singularity on its circle of convergence. Suppose that near $x=\alpha, f(x)$ has an expansion of the form

$$
f(x)=(1-x / \alpha)^{-s} g(x)+(1-x / \alpha)^{-t} h(x)
$$

where $g(x)$ and $h(x)$ are analytic at $x=\alpha, g(\alpha) \neq 0, s$ and $t$ are real, $s \neq 0,-1,-2, \cdots$, and either $t<s$ or $t=0$. Then

$$
a_{n} \sim \frac{g(\alpha)}{\Gamma(s)} n^{s-1} \alpha^{-n}
$$

STEP 18. In the series

$$
s(x)=a_{0}+a_{1}(\sigma-x)^{1 / 2}+a_{2}(\sigma-x)+\cdots
$$

obtained in Step 14, we must have $a_{1}=0$ if

$$
\lim _{x \rightarrow-\sigma} s^{\prime}(x)<\infty \text {. }
$$

Computing $s^{\prime}(x)$ from the functional relation obtained in Step 2 for $s(x)$ in terms of $S(x)$ and $S\left(x^{2}\right)$, it will be found that since

$$
\lim _{x \rightarrow-\sigma} S^{\prime}(x)(S(\sigma)-S(x))<\infty
$$

as shown in Step 15, we obtain the finiteness of $\lim _{x \rightarrow-\sigma} s^{\prime}(x)$.

STEP 19. Once $a_{1}=0$ has been established, the proof that $a_{3}>0$ and the evaluation of $a_{3}$ parallel the corresponding procedures for $b_{1}$ followed in Step 16.

STEP 20. Collecting alternate terms in the expansion for $s(x)$ around $x=\sigma$ we find that

$$
s(x)=(\sigma-x)^{3 / 2} c(x)+d(x)
$$

where $c(x)$ and $d(x)$ are analytic at $x=\sigma$ and $c(\sigma)=a_{3}$. As $\Gamma(-3 / 2)=$ $(4 / 3) \pi^{1 / 2}$ we can at once determine the asymptotic behavior of $s_{n}$ from Pólya's Lemma.

\section{Identity trees}

A free tree is unrooted. An identity tree is a tree for which the only automorphism is the identity. An identity rooted tree is a rooted tree for which the only automorphism preserving the root point is the identity. An identity 
plane tree is an identity rooted tree which is rooted at an endpoint. Note that the underlying (unrooted) tree of an identity rooted tree may not itself be an identity tree.

Let $U_{n}$ be the number of nonisomorphic identity planted trees on $n+1$ points, which is easily seen to be the number of nonisomorphic identity rooted trees on $n$ points. Let $u_{n}$ be the number of free identity trees on $n$ points. Thus in this section $U_{n}$ will play the role of $S_{n}$ and $u_{n}$ that of $s_{n}$.

Let $U(x)$ and $u(x)$ be the ordinary generating functions

$$
U(x)=\sum_{n=1}^{\infty} U_{n} x^{n}, \quad u(x)=\sum_{n=1}^{\infty} u_{n} x^{n} .
$$

Then $U(x)$ and $u(x)$ take the place of $S(x)$ and $s(x)$. Let $\mu$ be the radius of convergence of $U(x)$, viewed as a power series in the complex variable $x$. The place of $\sigma$ is taken by $\mu$.

Note that $U_{i} \geqq 1$ for all $i \geqq 1$. As an example, the result of rooting a path of length $i$ at one of its endpoints is an identity planted tree which is among those counted by $U_{i}$. Thus the sequence $U_{1}, U_{2}, \cdots$ satisfies the two simple suppositions stipulated at the end of Section 1.

We now develop the 20 steps of Section 2 as they apply to $U(x)$ and $u(x)$. Specific details are supplied when necessary to complete the verifications indicated for the various steps in Section 3.

StePs 1 AND 2. Harary and Prins (1959; page 155) derived the basic functional relations

$$
\begin{aligned}
& U(x)=x e^{U(x)-\frac{1}{2} U\left(x^{2}\right)+\frac{1}{3} U\left(x^{3}\right) \neq \cdots} \\
& u(x)=U(x)-\frac{1}{2} U\left(x^{2}\right)-\frac{1}{2} U^{2}(x) .
\end{aligned}
$$

The relation (1) for $U(x)$ is easily seen to be equivalent to

$$
U(x)=x \prod_{i=1}^{x}\left(1+x^{i}\right)^{U_{i}}
$$

Relation $\left(1^{*}\right)$ can be established in a direct manner since each factor $\left(1+x^{i}\right)^{U_{i}}$ serves to enumerate the ways of choosing identity branches of weight $i$ without repetition. We consider these branches to be taken at the point adjacent to the root, and multiply by $x$ to include this point in the count.

STEP 3. If $T_{n}$ is the number of rooted trees on $n$ points, then $T_{n} \geqq U_{n}$ for every $n$. Thus $\mu$ is at least as large as the radius of convergence $\rho$ of the series

$$
T(x)=\sum_{n=1}^{\infty} T_{n} x^{n}
$$

Pólya (1937) showed that $\rho>0$ by comparing $T(x)$ with the generating function 


$$
\frac{x}{2}(1-\sqrt{1-4 x})
$$

for planted plane trees, expanded around $x=0$, which gives $\frac{1}{4}$ as a lower bound for $\rho$. We conclude as in Section 3 that

$$
0<\rho \leqq \mu \leqq 1 .
$$

STEP 4. Since $U_{i} \geqq 1$ for all $i \geqq 1$, it is clear that $U(x)$ is strictly increasing on the real interval $[0, \mu)$. Since $\mu \leqq 1$, we have

$$
U(x) \geqq U\left(x^{2}\right) \geqq U\left(x^{3}\right) \geqq \cdots
$$

for $x$ in $[0, \mu)$. From (1) it follows that

$$
U(x) \geqq x e^{v(x) / 2}
$$

holds on this interval. As $x$ approaches $\mu$ from below, the left side is linear in $U(x)$ while the right side increases exponentially with $U(x)$. Thus $U(x)$ must be bounded as $x$ ranges over $[0, \mu)$, so $U(\mu)<\infty$. From $U(\mu)<\infty$ and $U_{i} \geqq 1$ for all $i \geqq 1$, we have $\mu<1$.

STEP 5. Define

$$
G(x, y)=-y+x e^{y-\frac{1}{2} U\left(x^{2}\right)+\frac{1}{3} U\left(x^{3}\right) \mp \cdots} .
$$

Then $G(x, y)$ is analytic at $(x, y)$ if $|x|<\mu^{1 / 2}$, and by (1) we have

$$
G(x, U(x))=0
$$

for $|x|<\mu$.

STEP 6. Equation (3) is extended to the circle of convergence $|x|=\mu$ as in Section 3.

STEP 7. It is concluded that

$$
G_{y}(\mu, U(\mu))=0
$$

exactly as in Section 3.

STEP 8. Differentiating $G(x, y)$ directly from its definition, one has

$$
G_{y}(x, y)=G(x, y)+y-1 \text {. }
$$

Putting $x=\mu$ and $y=U(\mu)$, this gives

$$
G_{y}(\mu, U(\mu))=U(\mu)-1
$$

in view of Steps 5 and 6. By Step 7, then, $U(\mu)=1$.

STEP 9. Substituting 1 for $U(\mu)$ in the relation

$$
G(\mu, U(\mu))=0
$$


justified in Step 7, we find

$$
\mu=e^{-1+\frac{1}{2} U\left(\mu^{2}\right)-\frac{1}{3} U\left(\mu^{3}\right) \pm}
$$

This can be used to determine $\mu$ to a high degree of accuracy from a sufficiently long initial segment of the numbers $U_{1}, U_{2}, U_{3}, \cdots$. These are readily computed by a recurrence relation derived directly from $\left(1^{*}\right)$. In Table 1 the results of such computations are presented.

The determination of $\mu$ is now illustrated in showing that $\mu<1 / 2$, a fact put to use below in Step 16. If the relation for $\mu$ is expressed in the product form $\left(1^{*}\right)$ it becomes

$$
\mu=\prod_{i=1}^{\infty}\left(1+\mu^{i}\right)^{-U_{i}} .
$$

If $\mu \geqq 1 / 2$ then a contradiction arises from the inequality

$$
\mu=\prod_{i=1}^{\infty}\left(1+\mu^{i}\right)^{-U_{i}} \leqq \prod_{i=1}^{3}\left(1+\mu^{i}\right)^{-U_{i}}=\frac{2}{3} \cdot \frac{4}{5} \cdot \frac{8}{9}<\frac{1}{2},
$$

which follows from $U_{1}=U_{2}=U_{3}=1$.

SteP 10, As in Step 8 for the case of $x=\mu$, we have

$$
G_{y}(x, U(x))=U(x)-1
$$

for $|x|=\mu$. As explained in Section 3, it follows that

$$
G_{y}(x, U(x)) \neq 0
$$

if $|x|=\mu$ and $x \neq \mu$.

STEP 11. As in Section 3, it is concluded that $x=\mu$ is the sole singularity of $U(x)$ on its circle of convergence.

STEP 12. If we differentiate (4) and simplify using (4) itself we find

$$
G_{y y}(x, y)=G(x, y)+y .
$$

Thus,

$$
G_{y y}(\mu, U(\mu))=1
$$

in view of Step 6.

STEPS 13, 14 AND 15. As in Section 3, we see that expansions of the form

$$
\begin{aligned}
& U(x)=1-b_{1}(\mu-x)^{1 / 2}+b_{2}(\mu-x)+b_{3}(\mu-x)^{3 / 2}+\cdots \\
& U(x)=a_{0}+a_{1}(\mu-x)^{1 / 2}+a_{2}(\mu-x)+a_{3}(\mu-x)^{3 / 2}+\cdots
\end{aligned}
$$

are valid in some neighborhood of $x=\mu$, and 


$$
b_{1}^{2} / 2=\lim _{x \rightarrow-\mu} U^{\prime}(x)(1-U(x)) .
$$

Step 16. By (3) we have

$$
\frac{d}{d x} G(x, U(x))=0
$$

for $|x|<\mu$. Defining

$$
Q(x)=-\frac{1}{2} U\left(x^{2}\right)+\frac{1}{3} U\left(x^{3}\right) \mp \cdots,
$$

we have

$$
-U^{\prime}(x)+U(x)\left(U^{\prime}(x)+Q^{\prime}(x)\right)+U(x) / x=0
$$

or

$$
U^{\prime}(x)(1-U(x))=U(x)\left(Q^{\prime}(x)+\frac{1}{x}\right) .
$$

Since $Q(x)$ has radius of convergence $\mu^{1 / 2}>\mu$, the term $Q^{\prime}(x)$ is bounded at $x=\mu$. Thus, taking the limit as $x$ approaches $\mu$ from below and applying (5),

$$
b_{1}^{2} / 2=\frac{1}{\mu}+Q^{\prime}(\mu),
$$

that is,

$$
b_{1}^{2} / 2=\frac{1}{\mu}\left[1-\mu^{2} U^{\prime}\left(\mu^{2}\right)+\mu^{3} U^{\prime}\left(\mu^{3}\right) \mp \cdots\right] .
$$

It remains to show from this that $b_{1}^{2} / 2>0$. Since $x U^{\prime}(x)$ is strictly increasing for $x$ in the interval $[0, \mu)$ and $0<\mu<1$, we find at once that

$$
\mu^{2} U^{\prime}\left(\mu^{2}\right)>\mu^{3} U^{\prime}\left(\mu^{3}\right)>\cdots .
$$

Thus we need only show

$$
1>\mu^{2} U^{\prime}\left(\mu^{2}\right) \text {. }
$$

It was established in Step 9 that $\mu<1 / 2$, so $i \mu^{i}<i 2^{-i}<1$ for $i \geqq 1$. Therefore

$$
\mu^{2} U^{\prime}\left(\mu^{2}\right)=\sum_{i=1}^{\infty}\left(i \mu^{i}\right) U_{i} \mu^{i}<U(\mu)=1 .
$$

The value $b$, calculated from (6) is contained in Table 1 .

STEP 17. As in Section 3 it now follows that

$$
U_{n} \sim \frac{b_{1}}{2}\left(\frac{\mu}{\pi}\right)^{1 / 2} n^{-3 / 2} \mu^{-n}
$$

STEP 18. By the expansion in Step 14, 


$$
u^{\prime}(x)=-\frac{1}{2} a_{1}(\mu-x)^{-1 / 2}-a_{2}-\frac{3}{2} a_{3}(\mu-x)^{1 / 2}-\cdots
$$

On the other hand

$$
u^{\prime}(x)=U^{\prime}(x)(1-U(x))-x U^{\prime}\left(x^{2}\right)
$$

results from differentiating (2). The last term is analytic at $x=\mu$ while the other term on the right was shown to be bounded in Step 15. Thus

and so $a_{1}=0$.

$$
\lim _{x \rightarrow-\mu} u^{\prime}(x)<\infty,
$$

STEP 19. Differentiating the series for $u(x)$ about $x=\mu$ again, we have

$$
u^{\prime \prime}(x)=\frac{3}{4} a_{3}(\mu-x)^{-1 / 2}+\cdots,
$$

where the remainder is bounded as $x$ approaches $\mu$. In Step 16 it was shown that

$$
U^{\prime}(x)(1-U(x))=U(x)\left(Q^{\prime}(x)+\frac{1}{x}\right)
$$

so from Step 18 we find

$$
u^{\prime}(x)=U(x)\left(Q^{\prime}(x)+\frac{1}{x}\right)-x U^{\prime}\left(x^{2}\right)
$$

Differentiating gives

$$
u^{\prime \prime}(x)=U^{\prime}(x)\left(Q^{\prime}(x)+\frac{1}{x}\right)+U(x)\left(Q^{\prime \prime}(x)-\frac{1}{x^{2}}\right)-U^{\prime}\left(x^{2}\right)-2 x^{2} U^{\prime \prime}\left(x^{2}\right) .
$$

Each term on the right is bounded as $x$ approaches $\mu$ from below except for $U^{\prime}(x)$. As seen in Step 16,

$$
\lim _{x \rightarrow-\mu} Q^{\prime}(x)+\frac{1}{x}=b_{1}^{2} / 2
$$

Since

$$
U^{\prime}(x)=\frac{b_{1}}{2}(\mu-x)^{1 / 2}+\cdots,
$$

the remainder being bounded near $x=\mu$, we have

$$
\frac{3}{4} a_{3}=\frac{b_{1}}{2} \cdot \frac{b_{1}^{2}}{2}, \text { or } a_{3}=b_{3}^{3} / 3 \text {. }
$$

STEP 20. As in Section 3, it follows that

$$
u_{n} \sim \frac{b_{1}^{3}}{4}\left(\frac{\mu^{3}}{\pi}\right)^{1 / 2} n^{-5 / 2} \mu^{-n}
$$




\section{Homeomorphically irreducible trees}

A tree is homeomorphically irreducible if no point has degree 2. Let $H_{n}$ be the number of nonisomorphic planted homeomorphically irreducible trees on $n+1$ points. In general $H_{n}$ will not be the number of rooted homeomorphically irreducible trees on $n$ points because the operation of attaching a new root to the old will increase by one the degree of the old root point. Let $h_{n}$ be the number of free homeomorphically irreducible trees of order $n$. Thus $H_{n}$ and $h_{n}$ will substitute for $S_{n}$ and $s_{n}$ in this section.

Let $H(x)$ and $h(x)$ be the ordinary generating functions

$$
H(x)=\sum_{n=1}^{\infty} H_{n} x^{n}, \quad h(x)=\sum_{n=1}^{\infty} h_{n} x^{n} .
$$

Then $H(x)$ and $h(x)$ take the place of $S(x)$ and $s(x)$. Let $\theta$ be the radius of convergence of $H(x)$, viewed as a power series in the complex variable $x$. Thus $\theta$ takes the place of $\sigma$.

Note that $H_{1}=1, H_{2}=0$, and $H_{n} \geqq 1$ for $n \geqq 3$. The latter can be seen by rooting any endpoint of the star $K_{1, n}$. Therefore the sequence $H_{1}, H_{2}, H_{3}, \cdots$ satisfies the two assumptions made with regard to $S_{1}, S_{2}, S_{3}, \cdots$ at the end of Section 1.

The remainder of this section consists of the 20 steps applied to $H(x)$ and $h(x)$. Details are supplied when necesssary to complete the verifications indicated for each step in Section 3, provided that they differ from the details presented for the corresponding step in Section 4.

STEPS 1 AND 2. The relations derived by Harary and Prins (1959; page $150)$ for homeomorphically irreducible trees can be simplified to the pair

$$
H(x)=\frac{x}{1+x} e^{H(x)+\frac{1}{2} H\left(x^{2}\right)+\cdots}
$$

$$
h(x)=(1+x) H(x)-\frac{1}{2}(1+x) H^{2}(x)+\frac{1}{2}(1-x) H\left(x^{2}\right) .
$$

STEP 3. We have $T_{n} \geqq H_{n}$ for all $n \geqq 1$, and so just as in Section 4 it follows that

$$
0<\rho \leqq \theta \leqq 1 .
$$

STEP 4. Again $H(x)$ is strictly increasing on the interval $[0, \theta)$. Relation (7) has the left hand side increasing as $H(x)$ and the right side as $e^{H(x)}$, so $H(x)$ must be bounded on this interval. Thus $H(\theta)<\infty$, and, recalling that $H_{i} \geqq 1$ for all $i \geqq 3$, we note that $\theta<1$.

\section{SteP 5. Define}




$$
J(x, y)=\frac{x}{1+x} e^{y+\frac{1}{2} H\left(x^{2}\right)+\frac{1}{3} H\left(x^{3}\right)+\cdots}-y .
$$

Then $J(x, y)$ is analytic at $(x, y)$ if $|x|<\theta^{1 / 2}$, and by (7),

$$
J(x, H(x))=0
$$

whenever $|x|<\theta$.

STEP 6. Equation (9) is extended to the circle of convergence $|x|=\theta$ just as in Section 3.

STEP 7. It is deduced that

as in Section 3.

$$
J_{y}(\theta, H(\theta))=0
$$

SteP 8. As in Section 4 it is seen that

$$
\begin{aligned}
J_{y}(x, y) & =J(x, y)+y-1, J_{y}(\theta, H(\theta))=H(\theta)-1, \text { and } \\
H(\theta) & =1 .
\end{aligned}
$$

STEP 9. The relation $J(\theta, H(\theta))=0$ can be written as

$$
\theta=\left\{-1+e^{1+\frac{1}{2} H\left(\theta^{2}\right)+\frac{1}{3} H\left(\theta^{3}\right)+\cdots}\right\}^{-1} .
$$

In Table 1 we present a good estimate for $\theta$ computed from this equation, as well as the initial segment of values of $H_{1}, H_{2}, \cdots$ which was needed.

SteP 10. Just as in Section 4 we have

$$
J_{y}(x, H(x)) \neq 0
$$

whenever $|x|=\theta$ but $x \neq \theta$.

STEP 11. As in Section 3 it follows that $\theta$ is the sole singularity of $H(x)$ on its circle of convergence $|x|=\theta$.

STEP 12. By differentiating (10) again it is found (exactly as in Section 4) that

$$
J_{y y}(\theta, H(\theta))=1 \text {. }
$$

STEPS 13, 14 AND 15. As in Section $3, \theta$ is a branch point of order 2, so that $H(x)$ and $h(x)$ have expansions near $x-\theta$ in powers of $(\theta-x)^{1 / 2}$, that is,

$$
\begin{aligned}
& H(x)=1-b_{1}(\theta-x)^{1 / 2}+b_{2}(\theta-x)+b_{3}(\theta-x)^{3 / 2}+\cdots \\
& h(x)=a_{0}+a_{1}(\theta-x)^{1 / 2}+a_{2}(\theta-x)+a_{3}(\theta-x)^{3 / 2}+\cdots
\end{aligned}
$$

In addition, $b_{1}$ satisfies the relation

$$
\frac{b_{i}^{2}}{2}=\lim _{x \rightarrow-\theta} H^{\prime}(x)(1-H(x)) .
$$


STEP 16. Differentiating (7) one finds

$$
H^{\prime}(x)(1-H(x))=H(x)\left(\frac{1}{x(1+x)}+P^{\prime}(x)\right)
$$

for $|x|<\theta$, where we use the notation

$$
P(x)=\frac{1}{2} H\left(x^{2}\right)+\frac{1}{3} H\left(x^{3}\right)+\cdots .
$$

In the limit $x \rightarrow^{-} \theta$ this implies, in view of Step 15, that

$$
\frac{b_{1}^{2}}{2}=\frac{1}{\theta(1+\theta)}+\theta H^{\prime}\left(\theta^{2}\right)+\theta^{2} H^{\prime}\left(\theta^{3}\right)+\cdots .
$$

It is immediately seen that $b_{1}^{2}>0$. The value of $b_{1}$ can be computed on the basis of (11), the value of $\theta$ obtained earlier and a segment values of $H_{1}, H_{2}, \cdots$. The result is presented in Table 1 .

STEP 17. It follows as in Section 3 that

$$
H_{n} \sim \frac{b_{1}}{2}\left(\frac{\theta}{\pi}\right)^{1 / 2} n^{-3 / 2} \theta^{-n} .
$$

STEP 18. Differentiating (8) gives

$$
h^{\prime}(x)=(1+x) H^{\prime}(x)(1-H(x))+H(x)-\frac{1}{2} H\left(x^{2}\right)-\frac{1}{2} H^{2}(x)+x(1-x) H^{\prime}\left(x^{2}\right),
$$

from which it is seen that

$$
\lim _{x \rightarrow-\theta} h^{\prime}(x)<\infty,
$$

so that $a_{1}=0$ as in Section 3 .

STEP 19. Much as in Section 4 the identity of Step 16 can be substituted into that of Step 18; upon differentiating again one finds

$$
\begin{aligned}
h^{\prime \prime}(x)=H^{\prime}(x)\left[\frac{1}{x}+\right. & \left.(1+x) P^{\prime}(x)+1-H(x)\right]+H(x)\left[\frac{1}{x}+(1+x) P^{\prime}(x)\right]^{\prime} \\
+ & {\left[x(1-x) H^{\prime}\left(x^{2}\right)-\frac{1}{2} H\left(x^{2}\right)\right]^{\gamma} }
\end{aligned}
$$

All terms are bounded as $x \rightarrow^{-} \theta$ except for $H^{\prime}(x)$, and in particular

$$
\lim _{x \rightarrow-\theta}\left[\frac{1}{x}+(1+x) P^{\prime}(x)+1-H(x)\right]=(1+\theta) \frac{b_{1}^{2}}{2} .
$$

As in Section 4 it follows that

$$
\frac{3}{4} a_{3}=\frac{b_{1}}{2} \cdot(1+\theta) \frac{b_{1}^{2}}{2}, \text { or } a_{3}=\frac{b_{1}^{3}}{3}(1+\theta) .
$$


Then $a_{3}>0$ follows from $\theta>0$ and $b_{1}>0$.

Step 20. As in Section 3 it is concluded that

$$
h_{n} \sim \frac{b_{1}^{3}(1+\theta)}{4}\left(\frac{\theta^{3}}{\pi}\right)^{1 / 2} n^{-5 / 2} \theta^{-n} .
$$

\section{Tree-like Blocks}

Our final example is a family of graphs whose tree-like properties are a bit less obvious. It will be necessary to prove a lemma first which will demonstrate that the graphs in question correspond to certain "colored" trees. Then we may enumerate these particular colored trees in a manner similar to the one used by Riordan (1957) to count unrestricted colored trees. Only then can the asymptotic analysis be carried out.

In an attempt to extend the successful reconstruction of trees to that of blocks, it recently has been shown that those blocks having a point whose removal leaves a tree are in fact reconstructible. Let us call such blocks tree-like. The principal conclusion of our asymptotic analysis is that the fraction of all blocks which happen to be tree-like quickly decreases to $\mathbf{0}$ as the number of points increases. Thus, unfortunately, this effort has not significantly dented the vast stockpile of still unreconstructed blocks.

We begin by transforming the problem. If $G$ is a tree-like block, then $G$ has a point $v_{1}$ whose removal leaves the tree $T_{1}=G-v_{1}$. We proceed to color the points of $T_{1}$, coloring point $w$ red if $w$ was adjacent to $v_{1}$ and blue otherwise. Note that this coloring is not completely arbitrary, for, since $G$ is a block, every endpoint must be red. Clearly the colored tree $T$, uniquely specifies $G$, for we may replace $v_{1}$ and rejoin it to all the red points. However, it is not immediately obvious that $T_{1}$ has been determined uniquely, for $G$ may have had other points $v_{2}, v_{3}, \cdots$ whose removal produced different trees $T_{2}, T_{3}, \cdots$. We now show that all these colored trees are identical.

Lемма. If $G$ is a block with points $v_{1}$ and $v_{2}$ whose removal leaves colored trees $T_{1}$ and $T_{2}$ then $T_{1}$ and $T_{2}$ are isomorphic colored trees.

Proof. Clearly $v_{1}$ and $v_{2}$ must have the same degree, for each tree has the same number of lines. Let $d$ be the degree of $v_{1}$ and $v_{2}$ in $G$, and let $\varepsilon=1$ if $v_{1}$ and $v_{2}$ are adjacent and 0 otherwise. Then $v_{2}$ has degree $d-\varepsilon$ in $T_{1}$, and so $T_{1}$ has at least $d-\varepsilon$ endpoints. But $v_{1}$ has exactly $d-\varepsilon$ lines joining it to $T_{1}$, and so these lines must be joined precisely to those endpoints mentioned. Thus, $T_{1}$ must have no other endpoints, and only the endpoints may be red. We immediately observe that $T_{1}$ and $T_{2}$ must be isomorphic colored trees.

This lemma permits us to count 2-colored trees with only red endpoints instead of tree-like blocks. We proceed with this point of view. For conveni- 
ence, we henceforth refer to such trees as blocky. Let $C(x)$ and $c(x)$ denote the generating functions for planted and free blocky trees, and let $\gamma$ be the radius of convergence. Note that since the planted path of order $n+1$ may be colored in $2^{n-1}$ ways, we have $C_{n} \geqq 2^{n-1}$ for all $n \geqq 1$. As in the previous two sections, we shall present only those details which are necessary to carry out the 20-step algorithm.

STEP 1. The relation for $C(x)$ differs from the one for ordinary planted trees in two respects. First of all, the point adjacent to the plant can be colored in two ways, so we insert a factor of 2 . However, in the special case when there is only one line, we must color this point red, and so we have overcounted this term as $2 x$. To correct for this, we simply subtract $x$ to obtain

$$
C(x)=2 x e^{C(x)+C\left(x^{2}\right) / 2+C\left(x^{3}\right) / 3+\cdots}-x .
$$

STEP 2. The number of rooted blocky trees is basically the number of planted ones, except that we have overcounted when the new root is also a plant, for then it must be red. Thus, rooted blocky trees are counted by $(1-x) C(x)$. This may be used in the analog of Otter's equation to obtain the equation for free blocky trees:

$$
c(x)=(1-x) C(x)-\frac{1}{2} C^{2}(x)+\frac{1}{2} C\left(x^{2}\right) .
$$

STEP 3. Since $C_{n}>2^{n-1}$, we may conclude that $C(0.5)$ is infinite, so $\gamma \leqq 0.5$. Moreover, $C_{n}<T_{n} 2^{n}$ since, as a crude estimate, every point of each rooted blocky tree can be colored in at most two ways. Thus, recalling from Step 3 of Section 4 that the radius of convergence $\rho$ of $T(x)$ is at least 0.25 , we have

$$
0.125 \leqq \gamma \leqq 0.5 \text {. }
$$

STEP 4. Since $C(x)>0$ for $0<x<\gamma$, we estimate equation (1) by

$$
C(x) \geqq 2 x e^{C(x)}-x .
$$

Thus, $C(x)$ is bounded as $x$ approaches $\gamma$, and so $C(\gamma)$ is finite. In this instance, we already know $\gamma \leqq 0.5$ so we need not now observe that $\gamma$ is strictly less than 1.

Step 5. Define

$$
K(x, y)=-x-y+2 x e^{y+C\left(x^{2}\right) / a+c\left(x^{3}\right) / 3+\cdots}
$$

Then $K(x, y)$ is analytic at $(x, y)$ if $|x|<\gamma^{1 / 2}$ and by (12)

$$
K(x, C(x))=0 \text { whenever }|x|<\gamma \text {. }
$$

STEPS 6 AND 7. These steps are the same as in previous sections. 
STEP 8. Differentiating (15) directly, we have

$$
K_{y}(x, y)=K(x, y)+x+y-1 .
$$

From Step 7 we conclude that

$$
K_{y}(\gamma, C(\gamma))=0=\gamma+C(\gamma)-1 .
$$

Note that this time $C(\gamma)=1-\gamma$ instead of simply 1 as in the other examples we have considered.

STEP 9. The equation $K(\gamma, C(\gamma))=0$ from Step 6 can now be rewritten as

$$
1 / 2 \gamma=e^{1-\gamma+C\left(x^{2}\right) / 2+C\left(x^{3}\right) / 3+\cdots}
$$

In this form, we may obtain a good estimate for $\gamma$ using the first several coefficients $C_{1}, C_{2}, \cdots$.

STEPS 10-15. These steps are similar to those in the two previous sections, only this time the expansion for $C(x)$ near $x=y$ takes the form

$$
C(x)=1-\gamma-b_{1}(\gamma-x)^{1 / 2}+b_{2}(\gamma-x)+b_{3}(\gamma-x)^{3 / 2} \cdots .
$$

Consequently, $b_{1}$ satisfies the relation

$$
\frac{b_{1}^{2}}{2}=\lim _{x \rightarrow-\gamma} C^{\prime}(x)(1-\gamma-C(x)) .
$$

STEP 16. Differentiating (12) and setting

we obtain

$$
R(x)=\sum_{i=2}^{\infty} C\left(x^{i}\right) / i,
$$

$$
C^{\prime}(x)(1-x-C(x))=C(x) / x+(x+C(x)) R^{\prime}(x) .
$$

Taking the limit as $x \rightarrow^{-} \gamma$ yields

$$
\frac{b_{1}^{2}}{2}=\frac{1-\gamma}{\gamma}+R^{\prime}(\gamma)
$$

Finally, this expression can be used to evaluate $b_{1}$ in terms of $\gamma$ in the form

$$
\frac{b_{1}^{2}}{2}=\frac{1}{\gamma}-1+\sum_{n=1}^{\infty} \frac{n C_{n} \gamma^{2 n-1}}{(1-\gamma n)}
$$

The resulting value of $b_{1}$ is presented in Table 2 .

STEP 17. As in Sections 3 and 4 , it follows that

$$
C_{n} \sim \frac{b_{1}}{2}\left(\frac{\gamma}{\pi}\right)^{1 / 2} n^{-3 / 2} \gamma^{-n}
$$


STEP 18. Differentiating (13) gives

$$
c^{\prime}(x)=C^{\prime}(x)(1-x-C(x))-C(x)+x C^{\prime}\left(x^{2}\right),
$$

from which we see that

so that $a_{1}=0$.

$$
\lim _{x \rightarrow-\gamma} c^{\prime}(x)<\infty,
$$

STEP 19. Substituting from equation (17) of Step 16 into equation (19) above and then differentiating a second time yields

$$
\begin{aligned}
c^{\prime \prime}(x) & =C^{\prime}(x)\left(\frac{1}{x}-1+R^{\prime}(x)\right)+R^{\prime}(x)-\frac{C(x)}{x^{2}} \\
& +(x+C(x)) R^{\prime \prime}(x)+C^{\prime}\left(x^{2}\right)+2 x^{2} C^{\prime \prime}\left(x^{2}\right) .
\end{aligned}
$$

The only unbounded term as $x$ approaches $\gamma$ from below is $C^{\prime}(x)$, and so we use equation (18) to obtain

$$
\frac{3}{4} a_{3}=\frac{b_{1}}{2}\left(\frac{b_{1}^{2}}{2}\right),
$$

just as in Section 4. Thus, $a_{3}=b_{1}^{3} / 3$.

STEP 20. As in Section 3, we conclude that

$$
c_{n} \sim \frac{b_{1}^{3}}{4}\left(\frac{\gamma^{3}}{\pi}\right)^{1 / 2} n^{-5 / 2} \gamma^{-n}
$$

\section{References}

E. A. Bender (to appear), 'Asymptotic methods in enumeration' .

F. Harary and E. M. Palmer (1973), Graphical Enumeration (Academic Press, New York, 1973).

F. Harary and G. Prins (1959), 'The number of homeomorphically irreducible trees, and other species', Acta Math. 101, 141-162.

E. Hille (1959), Analytic Function Theory, Vol. I (Ginn, Boston, 1959).

R. Otter (1948), 'The number of trees', Ann. of Math. 49, 583-599.

G. Pólya (1937), 'Kombinatorische Anzahlbestimmungen für Gruppen, Graphen und chemische Verbindungen', Acta Math. 68, 145-254.

J. Riordan (1957), 'The number of labelled colored and chromatic trees', Acta Math. 97, 211-225.

E. T. Whittaker and G. N. Watson (1943), A Course of Modern Analysis (Fourth Edition, Cambridge Univ. Press, Cambridge, 1943).

University of Michigan

Ann Arbor, Michigan, U.S.A.

University of Newcastle

New South Wales, Australia

Michigan State University

East Lansing, Michigan, U.S.A. 
Table 1

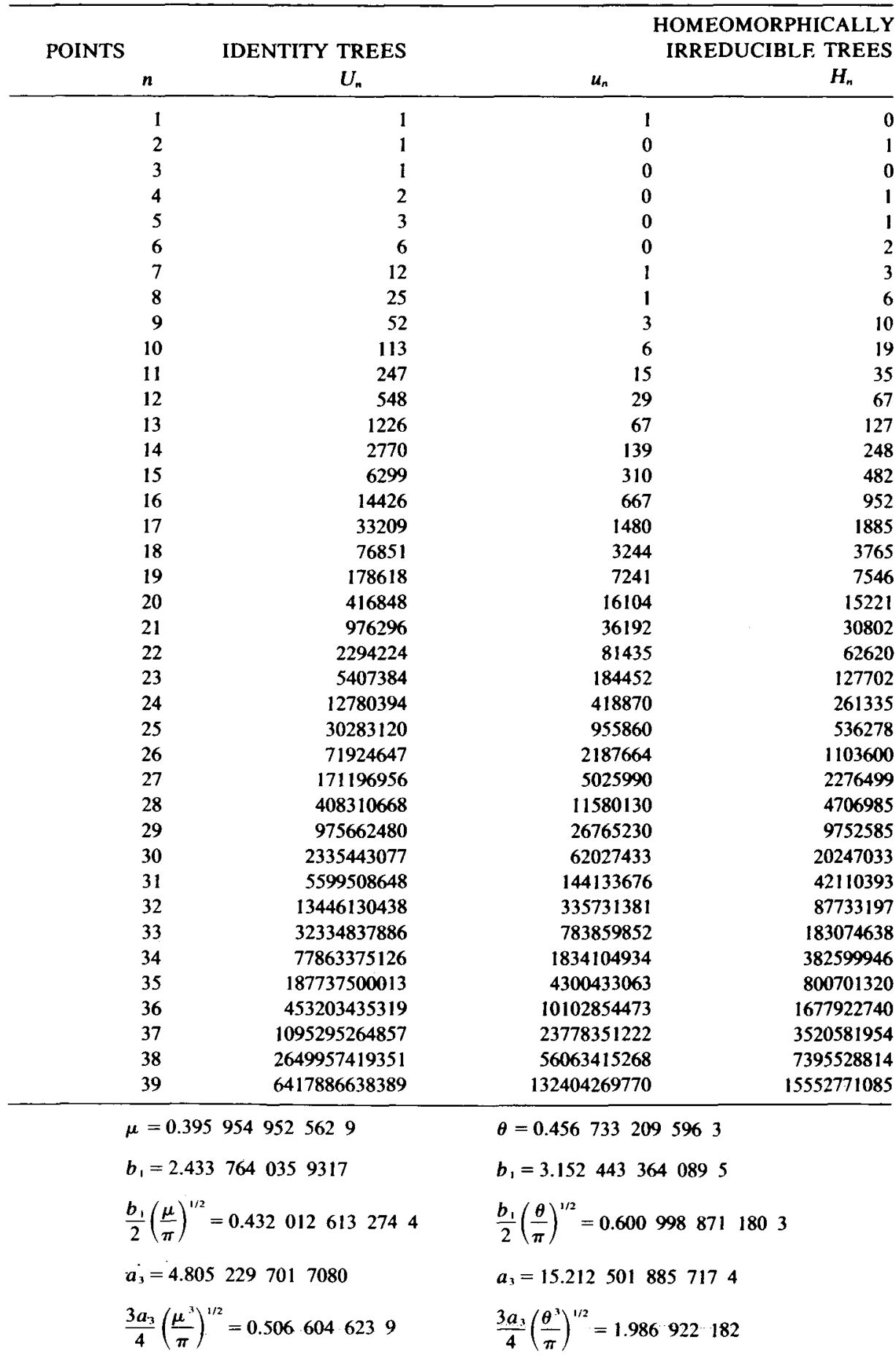


Table 2

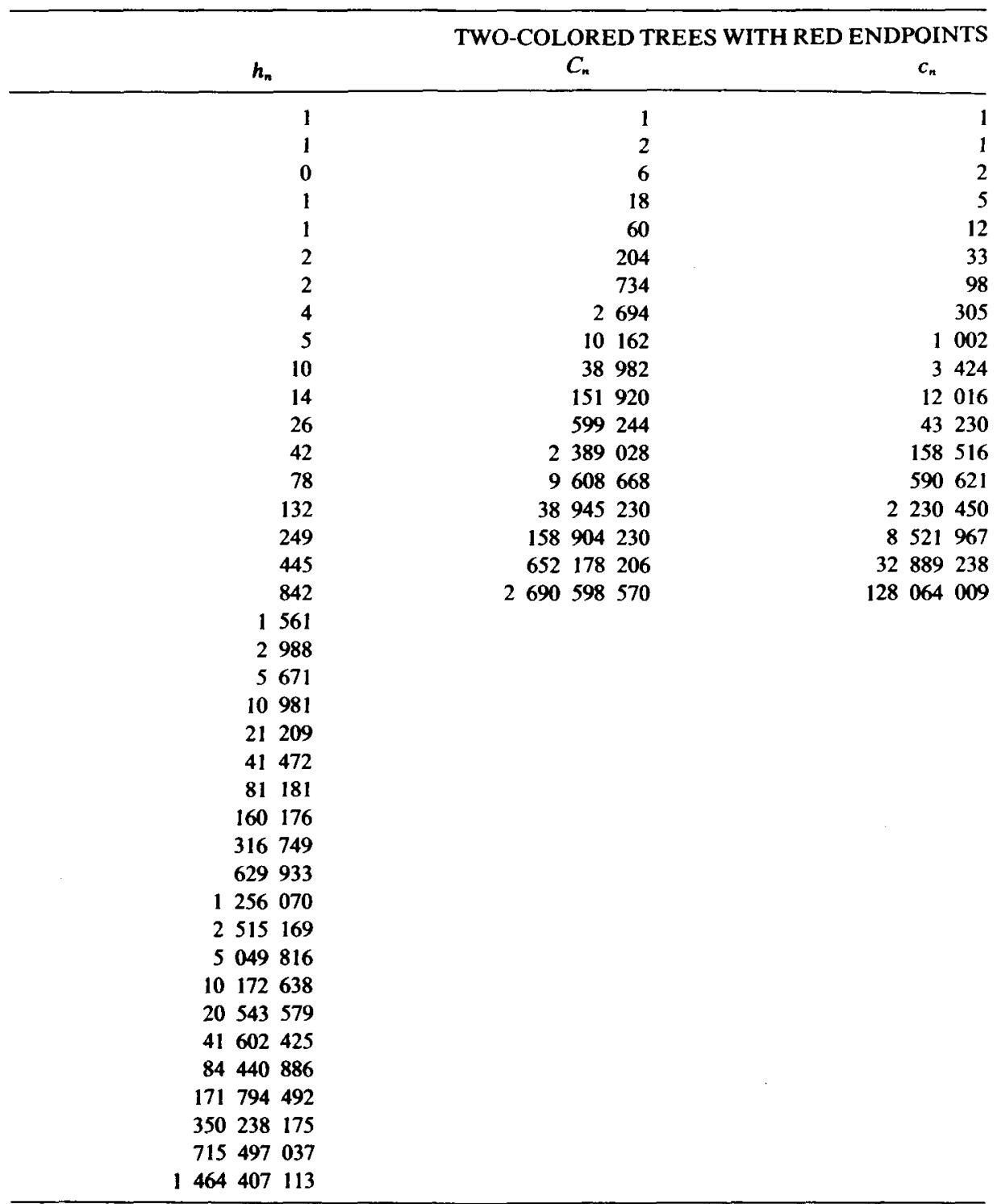

$$
\begin{aligned}
& \gamma=0.2225111688 \\
& b_{1}=2.509669780 \\
& \frac{b_{1}}{2}\left(\frac{\gamma}{\pi}\right)^{1 / 2}=0.3339546974 \\
& a_{3}=5.269003521 \\
& \frac{3 a_{3}}{4}\left(\frac{\gamma^{3}}{\pi}\right)^{1 / 2}=0.2340143763
\end{aligned}
$$

